\title{
Disruption and adaptation of urban transport networks from flooding
}

\author{
Maria Pregnolato $^{1, a}$, Alistair Ford ${ }^{1}$ and Richard Dawson ${ }^{1}$ \\ ${ }^{1}$ School of Civil Engineering and Geosciences, Newcastle upon Tyne, NE1 7RU, UK
}

\begin{abstract}
Transport infrastructure networks are increasingly vulnerable to disruption from extreme rainfall events due to increasing surface water runoff from urbanization and changes in climate. Impacts from such disruptions typically extend far beyond the flood footprint, because of the interconnection and spatial extent of modern infrastructure. An integrated flood risk assessment couples high resolution information on depth and velocity from the CityCAT urban flood model with empirical analysis of vehicle speeds in different depths of flood water, to perturb a transport accessibility model and determine the impact of a given event on journey times across the urban area. A case study in Newcastle-upon-Tyne (UK) shows that even minor flooding associate with a 1 in 10 year event can cause traffic disruptions of nearly half an hour. Two adaptation scenarios are subsequently tested (i) hardening (i.e. flood protection) a single major junction, (ii) introduction of green roofs across all buildings. Both options have benefits in terms of reduced disruption, but for a 1 in 200 year event greening all roofs in the city provided only three times the benefit of protecting one critical road junction, highlighting the importance of understanding network attributes such as capacity and flows.
\end{abstract}

\section{Introduction}

In recent years flooding has become increasingly disruptive in Europe and across the world. There have been a number of extremely disruptive flood events in the UK in recent years, with Cumbria being particularly badly affected (2015 and 2016), and widespread flooding in past years (2005 and 2009 in Cumbria, 2007 in Yorkshire, 2012 all over UK) [1].

Cities are often particularly vulnerable to such hazardous events, since they concentrate people and assets in highly-connected and complex systems. This vulnerability is increasing in many locations, especially under pressure from climate change and urbanisation. Infrastructure systems are the backbone of urban environments, and their efficient operation relies a number of interdependencies between networks such as transport, communication or electricity [2]. Reduced performance of such can lead to great disruption to urban systems, with interconnections and interdependencies leading to knock-on effects beyond the network which is initially affected [3].

There is increasing awareness in many communities of changes to the frequency of flood events and the inadequacy of current policies and flood defences. Improving the resilience of infrastructure systems to this ever-increasing threat, through a number of possible interventions, is essential to ensure the continued habitability of urban areas. This study aims to assess flooding impacts for a case study in UK and test several urban infrastructure adaptation measures to assess their potential for improving urban resilience.

\footnotetext{
${ }^{\mathrm{a}}$ Corresponding author: m.pregnolato@ncl.ac.uk
}

\section{Background and motivation}

According to Swiss RE [4] 40 major catastrophic events took place in Europe in 2013, including two of the most costly in the world ( $\$ 16.5$ billion of damage for the Germany Czech Republic floods; $\$ 4.8$ billion for hailstorms in France and Germany). The UK suffered multiple extreme events, with $£ 3.2$ billion of economic losses in 2007 for floods in England and £276 million for the Cumbria floods in 2009 [5]. Considering all the possible source of flooding (fluvial, coastal, surface and groundwater), floods today contribute for $£ 1.3 \mathrm{~b}$ of expected annual damages in UK only [6]. Additionally, such damages are projected to increase in the future.

Most contemporary urban environments are characterised by a high percentage of impermeable surfaces (asphalt, tarmac, concrete), of which $40-50 \%$ is made up by roofs [7]. These environmental characteristics lead high volumes of surface runoff during rainfall events, forcing often out-dated drainage systems to cope with large quantities of additional water. Newcastle-upon-Tyne (North-East of England) is an example of a highly-impermeable city $(90 \%$ of surfaces are impermeable), and has been adopted as case study for this research due to availability of data and good stakeholder relations.

Traditional methods of flood protection and mitigation include strategies of hard engineering, like dams or dikes. Such measures can be effective, but are costly to build and are designed for a specific return period of event. Given the non-stationary nature of 
climate and the associated changes in frequency and magnitude of flooding events, this can be a great limitation. Alternative methods of "green engineering" include soft measures of urban interventions, like rain gardens, swales and ponds; these strategies are named BGI (Blue-Green Infrastructure) or, more generally, SUDs (Sustainable Drainage Systems). These strategies differ from conventional engineering approaches in that they provide multiple co-benefits alongside flood risk alleviation (e.g. habitat protection and biodiversity).

The effective operation of urban transport systems is essential for a city's businesses, employees, and economic competitiveness. Any damage to these systems could lead to severe and far-reaching consequences and further exacerbating effects, such as congestion, are known to lead to even greater economic costs [8]. In addition, transport networks are of fundamental importance during emergencies, with the resilience of such networks being vital to communication and emergency movements of people and material. In order to understand the role of green engineering solutions in reducing flood risk to these networks and systems, the impact of pluvial flooding in urban areas has been examined in this study.

The aim of the research, therefore, was to assess how urban environments can be impacted by extreme rainfall events and which strategies could help to better protected them from present and future flooding. In order to address this aim, four main aspects were addressed: $i$ ) testing damage that extreme rainfall can cause, from the point of view of traffic disruptions; ii) presenting emerging trends of urban adaptation interventions, based on green engineering; iii) testing urban strategies to enhance road network response to flooding; iv) facilitating a transition to sustainable planning with design principles of green/blue features.

\subsection{Improve urban stormwater management though infrastructure adaptation}

Many cities are proposing the use of green infrastructure to address urban extreme weather events, since they provide multiple co-benefits alongside their intended adaptation potential. The evidence for their effectiveness in urban adaptation to extreme events, however, is ambiguous [9]. This study will examine the effectiveness of green infrastructure, alongside other adaptation measures, in reducing the risk of disruption to transport from extreme rainfall.

\subsubsection{Green Infrastructure}

Alternative flood management techniques (for example green spaces and roofs, roof or underground storage, or permeable surfaces) can play a part in reducing the impact of floods, replacing traditional "hard" measures such as flood walls and embankments, to face a "new era" of flood risk [10]. The report into the UK floods of 2007 [11], highlights a number of examples of such innovative solutions, including garden without impervious surfaces, small-scale buildings on floodplains, systems to allow water from roofs and streets to seep and be filtered into the ground, or permeable car parks.

The primary benefits of such solutions are with regard to stormwater management and flood alleviation, by using their capacity to retain a volume of water and increasing the retention time. In fact, when the total storm water runoff is reduced, peak flows decrease as well. Secondary benefits include environmental aspects, such air quality, and mitigation of pollution, biodiversity, and temperature control. In addition, the quality of urban spaces is enhanced by reducing noise, regulating the microclimate, and adding amenity.

In this study the option of green roofs is tested, in parallel with an option of hard engineering (improving the resilience of critical links in the transport network), to assess their effectiveness in reducing risks to transport movements. Green roofs are particularly suitable for attenuating and delaying water into drainage after the peak of a rainfall event. Moreover, they serve to recycle water for irrigation. Studies have showed that the presence of vegetation on top of buildings can retain rainfall from $45 \%$ up to $78 \%$ [12], and can increase two or three times the roof lifespan. However, the effectiveness of such options is still questioned if applied to large-scale areas, such as across an entire city.

\section{Methodology}

A model to assess the impact of flood-related events on the urban transport network was developed, examining traffic disruptions due to flooding on roads. The framework on which the model is built upon is presented in Figure 1. This approach integrated hazard maps, output from an urban flood model, with the transport network model and a vulnerability analysis.

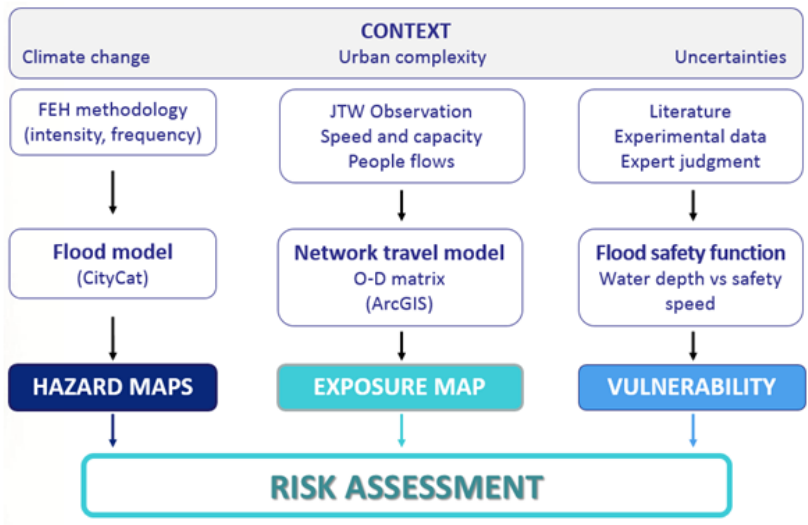

Figure 1. Methodological framework of the inter-disciplinary approach.

The preliminary step is to simulate multiple hazard scenarios, to obtain outputs in terms of floodwater depth in the city. A baseline transport scenario is then generated, by running the transport model under normal settings (i.e. speed limits imposed by UK traffic laws. Commuting flows (Journey-to-Work (JTW) trips) and routes are also determined between origin and destination (e.g. location of residence and location of employment). 
The second stage consists in running the model for a set of probabilistic hazard scenarios, to be compared to the baseline data. The outputs from the flood model (see section 4.1) are used to calculate impacts on the transport network; this impact is translated into speed reduction and consequent rerouting of the flows. A vulnerability curve (described in section 4.2) relating safe driving speed for cars to water depth has been developed to obtain this result. Thus, additional travel time due to flooding impacts can be computed. The delays, due to rerouting and speed reduction, are the metric of measurement for the impact, and can be converted into monetary terms using value of time [13].

By modifying parameters within the framework, different adaptation options can be tested. During flood simulation, the permeability of the soil can be modified, and roof or underground storage can be introduced to retain water volume. The network can be modified introducing more redundancy or varying link characteristics. Finally, commuting flows can be modified in order to explore alternative strategies, such as an increase in cycling to work or more working from home.

In this specific study, two options will be investigated: i) $100 \%$ green roofs, in order to assess the effectiveness of an extreme adaptation scenario; ii) hardening of a single road junction (node) or length of road (link), that is critical for the access to the city, in order to assess the effectiveness of hard engineering solutions.

The methodology presented here requires data and resources usually available for most metropolitan areas in Europe and the US, such as digital terrain models, census commuting data, and road network representations with attributes for speed and thus travel time. The software adopted was built using a Python framework and includes a hydrological model (CityCAT, see section 4.1) and GIS tools for spatial analysis.

\subsection{Limitations and assumptions}

The relationship between adverse weather, traffic flow, and congestion is known to be very hard to define [14-17]. Given that that cities are complex systems, problems are even more challenging when they are focused on networked systems and highly detailed assessments are very demanding, in terms of data collection and computation.

Micro-simulation and more advanced transport modelling techniques (e.g. dynamic traffic assignment) require large amounts of observed data and computational resources. The reduced-complexity, macro-scale, approach here does not consider the individual choices of each driver, but assumes they will try to select the lowest cost journey.

The model incorporates congestion effects (e.g. roads becoming busy), but does not account for traffic lights, queuing, and possible accidents (that are more likely during wet conditions). Modelling only considers JTW trips during general rush hours, without differentiation between morning or evening peaks.
Furthermore, the flood analysis was restricted to the city boundary of Newcastle-upon-Tyne. Journeys into and out of the city are considered, but flooding outside the city boundary which could have a knock on effect within the city limits, is not modelled here. Additionally, only flood depth is considered, whereas flood velocity is assumed minimal (as is the case for wind, visibility, driver behaviour, and other circumstances that can influence dynamics which are not examined).

Validation of this framework, to assess whether the model is close to be truly predictive, or should be limited to use in an exploratory manner, is an on-going process. However, traffic records from Newcastle City Council for the $28^{\text {th }}$ June 2012 urban flood in Newcastle show a good fit to the model, for that one event and further, smaller, events are being analysed.

Given these assumptions due to the nature of the macro-model, results should be compared relatively against each other rather than in absolute. Nevertheless, the analysis offers an integrated view on a complex problem, presenting a specific indication of flood impacts on transport network, and provides basis for further studies. Many of these limitations are being addressed in further work, as noted in the concluding section.

The techniques and data are readily available for many countries around the world so that the approach is transferable to other contexts. This methodology can be applied to present conditions as well as future scenarios including potential adaptation strategies, allowing the examination of impacts alongside socio-economic and climate change. Finally, whilst this study focuses on the flood risk to the road network, the framework can also be applied to other weather-related phenomena facilitating the systematic analysis of their direct and indirect impacts.

\section{Case Study}

The presented framework has been applied to a large-scale network in a specific demonstration case study. A preliminary analysis has been run in Tyne and Wear (in North East England, Fig. 2) to demonstrate how the analysis can be used to assess the disruptions to commuter journeys due to flooding. The analysis conducted is based on the comparison between pre-event and post-event travel times. The storm is assumed to fall only on Newcastle City Council area, but travel times are modelled for the wider Tyne and Wear transport region. Newcastle city centre has been adopted as a prototype case study, but other UK cities are currently being tested, including London. 


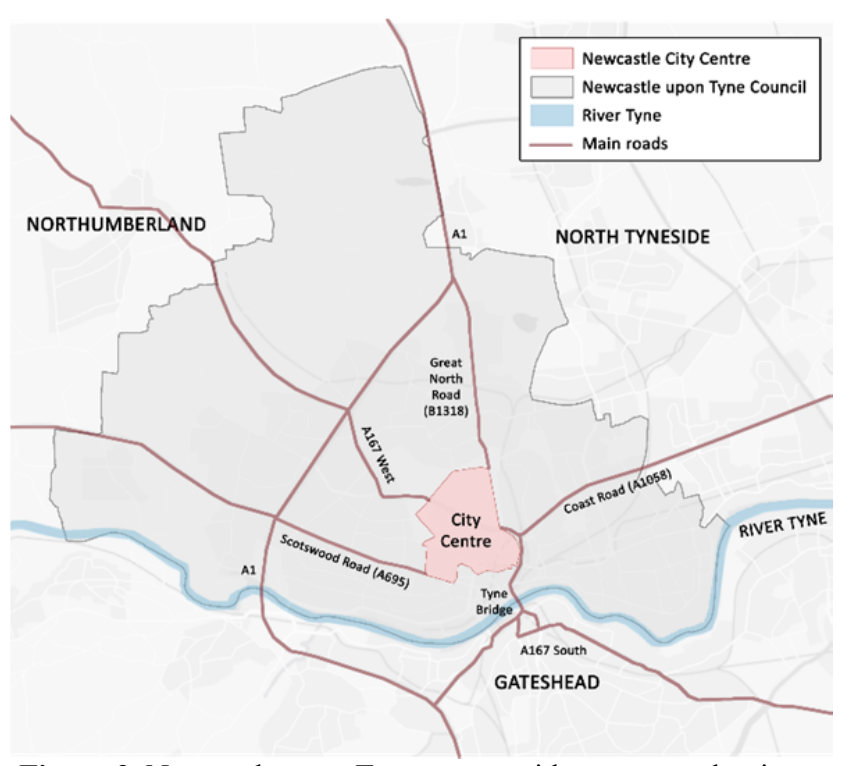

Figure 2. Newcastle upon Tyne extent with respect to the river Tyne position and the city centre.

Newcastle has a high flooding potential, with numerous historic events, together with high road density. The city centre is almost impervious (92\%) and there are no specific strategies for innovative drainage systems to cope with future rainfall extremes. These issues are not unique to the city, and it could be easily adopted as a prototype for medium cities in the UK for analysing pluvial floods [18].

In this study, seven scenarios (including the baseline where no flooding occurs) were analysed, against two different hazard profiles (10-years and 200years return period) and two urban flood risk management strategies (link hardening and green roofs). Tab. 1 offers a summary of the investigation.

\begin{tabular}{|c|c|c|}
\hline Label & Scenario & Return period \\
\hline NA_0 & No adaptation & No flood \\
\hline NA_10 & No adaptation & $10 \mathrm{ys}$ \\
\hline NA_200 & No adaptation & $200 \mathrm{ys}$ \\
\hline RE_10 & Harden road junction & $10 \mathrm{ys}$ \\
\hline RE_200 & Harden road junction & $200 \mathrm{ys}$ \\
\hline GR_10 & Green roof all buildings & $10 \mathrm{ys}$ \\
\hline GR_200 & Green roof all buildings & $200 \mathrm{ys}$ \\
\hline
\end{tabular}

Table 1. Overview of the simulated scenarios

\subsection{Characterising the flood hazard}

The hazard is represented by floodwater depth due to extreme rainfall and determined by simulating surface water flooding. Flood depth has been chosen as the most significant key metric in relation to transport network disruption, in accordance with the literature [19, 20].

Following the standard FEH methodology [21], floodwater depths were produced using the City Catchment Analysis Tool (CityCAT) software. CityCAT is a 2D hydrodynamic flood model based on the evaluation of infiltration of pervious areas. In recent years, Cloud computing has made possible the simulation of a large number of ensembles, allowing assessment of the uncertainty and variability of extreme rain events in the present and future conditions [22]. Simulations can be undertaken on current climatic conditions or future scenarios, based on the rainfall duration and return period.

Digital Terrain Models (DTMs) are utilized as inputs to the hazard modelling stage to generate the underlying topography, and topographic spatial data is used to describe the features of the built environment. For this UK case study Ordnance Survey MasterMap Data is employed. The inclusion of buildings, soil porosity, and other characteristic parameters allows more realistic simulation of flow paths in urban areas. This allows scenarios of present urban development to be tested, but scenarios of future development also allow the testing of future pathways (e.g. trends in urbanisation).

The spatial footprints of the simulated flood event produce a time series of hazard maps showing water depths. For this study, two different scenarios were chosen for initial testing prior to simulating a wider range of events in the probabilistic approach described above. The first scenario (A) is defined by the peak of a 10 -year return period event of 60 minutes duration; the second, more severe, scenario (B) being 200-year return period and 60 minutes duration. Fig. 3 shows the portion of network impacted by the two different flooding events. As expected, the more severe event disrupted a larger proportion of the transport network. A large increase is seen in the section of the network impacted by deeper water, with more than double the number of links affected by the deepest water (depth $>0.30 \mathrm{~m}$ ) and a 40 $60 \%$ increase in the proportion of the network flooded to a depth of $0.10 \mathrm{~m}$ to $0.30 \mathrm{~m}$.
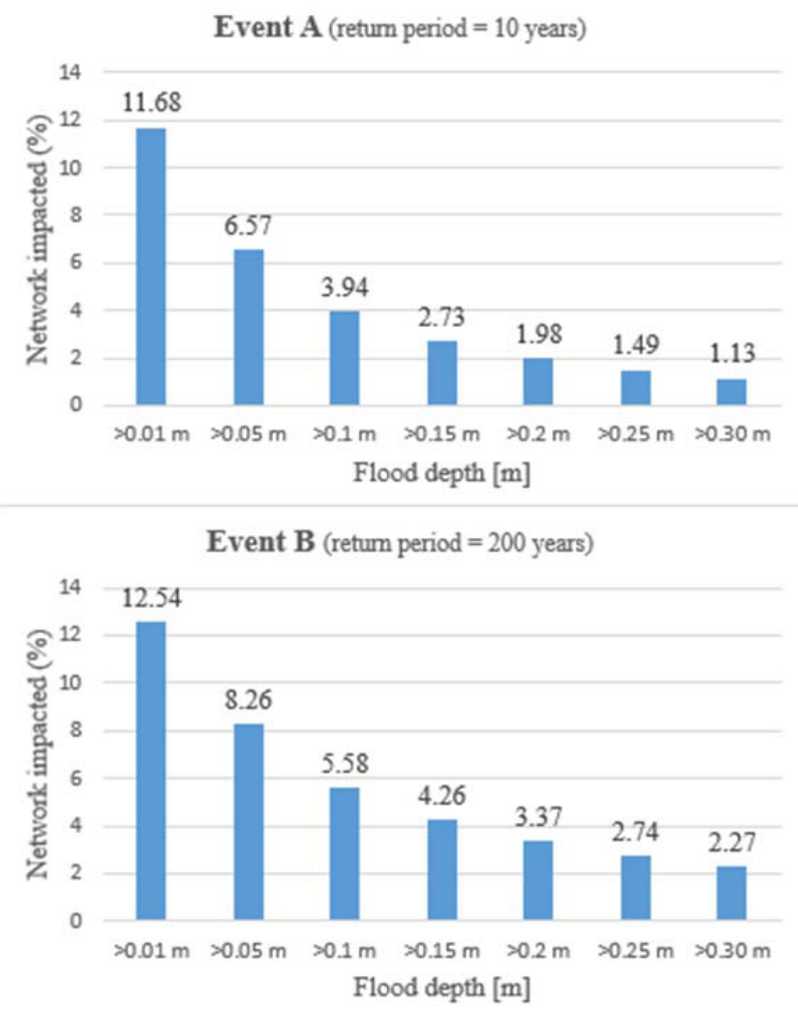

Figure 3. Histogram representing the percentage of network (in metres of length) affected by multiple flood depth, for hazard A and $\mathrm{B}$. 


\subsubsection{Adaptation simulation}

The model presented in this paper was used to assess the effectiveness of potential BGI in terms of green roofs in the urban area. The results presented in this paper consider a capacity of $5 \mathrm{~cm}$ of water depth per roof. The scenario imagines a hypothetical future city with $100 \%$ of roofs with rainwater storage in order to test the extreme limits of the effectiveness of this adaptation measure (although in reality such roofs could only physically be fitted to a small number of existing buildings). In this context, the hazards resulting from flooding scenarios (for example: event A, 10-years return period) are reduced by the implementation of the adaptation (Fig. 4).

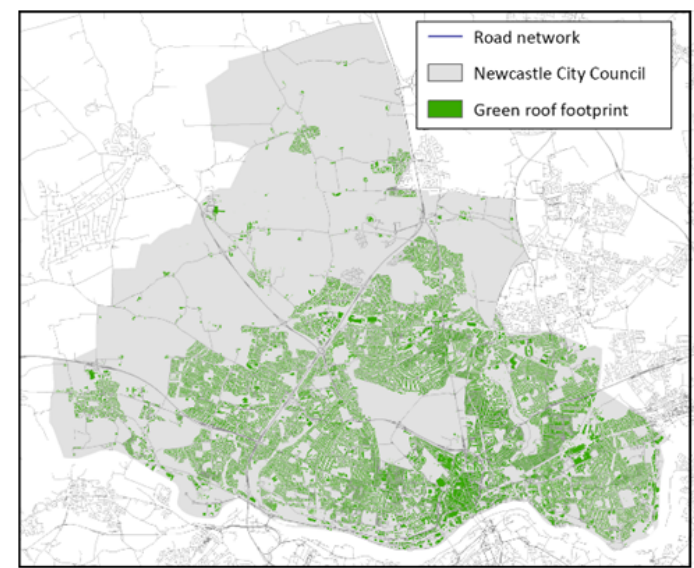

Figure 4. Building roofs footprint for Newcastle. For the ease of the analysis, $100 \%$ of roofs were considered as green roofs.

The roof storage, by delaying the release of rain water onto the surface, reduced both peak flow rates and total runoff volume of rainwater compared to the baseline simulation. As a source control mechanism in SUDs, green roofs can help reduce flash floods caused by intense rainfall events but their effectiveness for the most extreme rainfall events may be limited, especially during long wet spells [23].

\subsection{Characterising the flood impact}

As the study highlighted the impacts on the commuters in terms of disruption to their journeys, a model of network trips was developed using a Geographic Information System (ArcGIS). The model used a simple all-or-nothing trip assignment routine to load Journey-to-Work (JTW) observations from the 2011 UK census onto network models in GIS. The JTW trips are calculated as least cost routes between origin and destination location according to the shortest time of travelling.

By overlaying the CityCAT water depth onto the transport network, impacts in terms of increase travel time were assessed. If a link experienced a time delay due to the presence of water, the journey was recalculated according to the least-cost alternative route. Therefore, the disruption was calculated as extra time needed to achieve the journey.

\subsubsection{Vulnerability analysis}

Vulnerability curves represent a consistent method for flood risk assessment. However, little research explains in detail the methodology of their construction and their application [20]. This paper presents a simplistic, but functional curve developed by the authors, with a practical application in the case study.

In order to explore the impact of the hazard outputs with the spatial locations of the network, the floodwater depths simulated by CityCAT must be associated with safe travelling speed for cars into water (safety speed, i.e. the velocity considered safe for travelling through a specific water level) through a function. For this fundamental step, a depth-disruption function has been developed (see Figure 5).

The definition currently available in literature (e.g.: Penning-Rowsell E., Priest S. [24]) of flooding thresholds are limited to binary terms, e.g. declaring a road closed or open depending on the presence or absence of floodwater. To better represent the true impact, a set of curves have been developed (see Figure 5 for an example

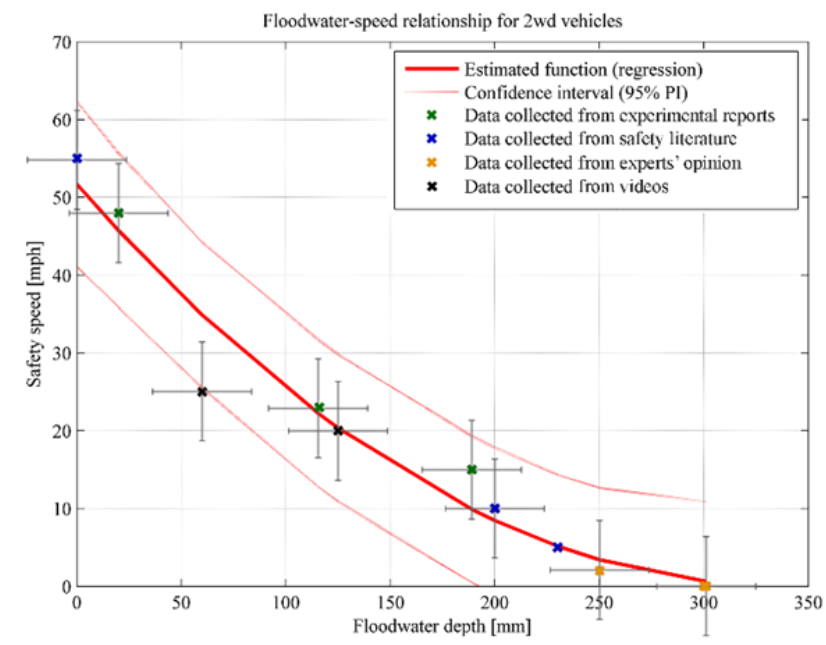

Figure 5. The transport function used for the vulnerability assessment.

for a road of 50mph speed limit) that relates water depth (between 0 and a critical flood depth where the road is impassable) to safe driving car speed [25].

The "key function" has been developed by combining data from experimental reports [26, 27], safety literature [28], experimental data [29], analysis of videos of cars driving through floodwater, and expert judgment (e.g. Automobile Association). The maximum threshold for safe driving is identified as $30 \mathrm{~cm}$ (the average sill height of a normal car) and therefore a link is assumed closed only when the limit of $30 \mathrm{~cm}$ is reached.

An upper and lower confidence interval are considered, to include uncertainties due to driving characteristic and behaviour (e.g. type of car, asphalt or tire, behaviour of the driver, visibility). Further research is needed to include uncertainties associated to each flood depth level and different type of cars.

The curve represents the safety function that relates floodwater depth and travelling speed during floods. By overlaying the hazard map with the road network, road segments that intersect flooded areas were defined and 
their modelled flood depth measured. For such segments, the speed was recalculated according to the presence of water on the link, according to the relation between flooding water depth and safety speed defined above through the safety function.

\subsubsection{City-scale transport modelling}

A large-scale traffic model has been developed to simulate urban flows for different hazard scenarios. After defining the baseline simulation with the first run of the model under normal settings, the model was rerun for multiple flooding scenarios, and the new outcomes were compared to the baseline. Travel times are calculated using the Dijkstra's algorithm (within ArcGIS), able to compute the shortest path between an origin and a destination in terms of time [30].

The final values, compared to the one computed without any disruption of the network, provided the difference as increased cost to the system. The impact of more frequent or extreme weather events, according to the hazard scenario considered, were assessed in terms of delays in time caused by perturbation of traffic flows (Tab. 2). A 'Person Minute Delay' value was calculated by multiplying the delay between each origin-destination pair with the observed commuting flow by car for that same pair.

\begin{tabular}{|c|c|c|}
\hline Label & $\begin{array}{c}\text { Total time of } \\
\text { delay }[\mathrm{min}]\end{array}$ & $\begin{array}{c}\text { Person } \\
\text { Minute Delay }\end{array}$ \\
\hline NA_0 & - & - \\
\hline NA_10 & 63006 & 783033 \\
\hline NA_200 & 119115 & 1438736 \\
\hline
\end{tabular}

Table 2. Results from the impact assessment

Such disruptions can be economically-evaluated since trips have an associated economic cost (e.g. lost work-time, lost sale, or production). In addition, there is extra travelling time and the cost of re-routing, dependant on the availability of alternative routes $[13,16,31]$.

\subsubsection{Critical links hardening}

In order to simulate the effects of grey adaptation (e.g. hard infrastructure), as a first step critical portions of the road network were identified. Subsequently, such hotspots were assumed to be made invulnerable to flooding during subsequent impact analysis (i.e. to simulate the effect of improved drainage).

On the basis of [32], critical links can be selected through a matrix of use and vulnerability. Vulnerability to flooding could be indicated by the simulated floodwater depth on corridors, whereas the maximum flux (e.g. cars/hour) was obtained by recorded data of traffic volume on the same links. This analysis was useful to quantify how severe the consequences could potentially be, due to the traffic level of the roads and flood depth on the corridor.

\subsection{Flood risk management}

In order to evaluate potential solutions to the problem of flooding on urban transport networks, two potential adaptation options were investigated. They were tested for both simulated hazard scenarios (sec. 4.1): the low-1 in 10 year, 60 minute, event and the 1 in 200 year, 60 minute, event. Commuting times were computed for the two scenarios without adaptations (RE and GR in Table 3 ), the two with adaptions and for both hazard simulations. Events RE_10 and RE_200 show the results for the hardening of critical links, and events GR 10 and GR_200 show the effects of green roofs. By comparing these values to the baseline NA_0, a measure of the benefits due to such adaptation strategies can be appreciated (Tab. 3).

\begin{tabular}{|c|c|c|c|}
\hline Label & $\begin{array}{c}\text { Total time } \\
\text { of delay } \\
{[\text { min }\rceil}\end{array}$ & $\begin{array}{c}\text { Person } \\
\text { Minute } \\
\text { Delay }\end{array}$ & $\begin{array}{c}\text { Benefit } \\
\%\end{array}$ \\
\hline BS_0 & - & - & - \\
\hline NA_10 & 63006 & 783033 & - \\
\hline NA_200 & 119115 & 1438736 & - \\
\hline RE_10 & 58960 & 689833 & $12 \%$ \\
\hline RE_200 & 115191 & 1343612 & $6 \%$ \\
\hline GR_10 & 48965 & 583309 & $25 \%$ \\
\hline GR_200 & 97262 & 1184125 & $18 \%$ \\
\hline
\end{tabular}

These results showed that significant delays can be measured in spite of simple assumptions about network behaviour (see section 3.1). The improvement in the number of people affected by flooding shows that adaptation interventions can support the maintenance of network performance during hazards. The hard intervention resulted less effective in lessening the impact, for both the hazard scenarios. However, green intervention was considered had an extreme nature (i.e. $100 \%$ of all roofs), implying impossible cost of intervention. Nevertheless, the green measure also provides a series of co-benefits which are not provided by grey infrastructure strategies. Indeed, the beneficial role of green roofs should not be focused within the scope of runoff management only, but consider the number of the associated benefits during decision-making (e.g. their role during extreme temperature events).

A small number of adaptation options are presented in this paper, but this methodology can be applied to test a complete portfolio through adjustments of land use and building characteristics in CityCAT alongside network interventions. Comparison between scenarios can determine the cost-effectiveness of the solutions considered, in association with a specific hazard intensity. This model could be considered a useful tool to help decision-makers assess the effectiveness of such measures and determine how best to include them within city council policies and plans.

\subsubsection{Discussion of results}

Two different adaptation options were tested, from "hard engineering" and "green engineering" respectively, alongside a low- and high-profile flooding events. These hazard maps and urban measures were combined, addressing six scenarios of flooding and adaptation.

Although both strategies resulted in a betterment of the network performance in case of disruption, for the 
city of Newcastle considering $100 \%$ of green roofs showed the greatest overall benefit. Although results demonstrated a positive contribution, the practicality of green roofs at city-scale is questionable. Adaptation effectiveness varies according to local context and conditions, in the case of green roofs it is highly unlikely that $100 \%$ of buildings could be retrofitted with a green roof due to structural limitations or other issues. Furthermore, the percentage of water retained can vary consistently, depending from roof structure, vegetation type, and rainfall magnitude. Finally, consideration of pre-existing soil level of saturation due to previous rainfall events or storms cluster has not been considered.

However, the hardening of a single critical part of the road network achieved $50 \%$ and $30 \%$ of the benefits of the green roof strategy. This highlights the importance of understanding the structure, capacity, connectivity, flows and other attributes of the transport network. When finances are limited, identifying key nodes and links on the road network can have a disproportionate benefit.

Nevertheless, the study highlights the importance of considering green infrastructure as part of a more integrated urban flood risk management. Future approaches should focus not only on traditional measures of hard interventions, but look at multi-faceted approach to planning, design and management of public spaces, as investments to mitigate storm impact and improve urban quality. A combination of carefully targeted hard and soft engineering investments would deliver the widest benefit in the face of uncertainties and complexities of urban flood risk.

\section{Conclusion}

The increasing number and impact of flooding disasters highlight the important role that the concepts of risk and resilience play for both current-day and future society.

This study detailed an integrated analysis investigating the flooding impact on free flow speed on urban road networks, delivers of key service for the community. The proposed methodology combined climate simulations and spatial representations together with a safety speed function. By overlaying in GIS spatial data regarding exposure, hazard thresholds from CityCAT, and a vulnerability curve, different levels of disruptions to commuting journeys on road networks were evaluated.

Newcastle-upon-Tyne (UK) was adopted as prototype to investigate the disruption on urban traffic due to flooding, in the form of time delays to commuter journeys caused by reduction in free flow speed and rerouting. As a first stage, two hazard scenarios were presented with differing magnitudes, to test combination of severity and intensity.

The results showed significant delays in traveling journeys, even for the low impact events ( 1 in 10 year return period). Two adaptation options (critical node hardening and green roofs) were tested and both showed substantial benefits to travel times. Whilst the urban greening strategy provided the greatest benefits, a single intervention to protect a road junction from flooding provided disproportionate benefits. As financial resources are typically limited it is crucial to understand the nature of flows along, and properties of, the road network in order to prioritise key assets. Balancing well located engineering interventions with urban greening will provide the most effective flood risk reduction.

Further work will analyse (i) a wider range of current and future rainfall intensities and durations, (ii) a wider range of urban flood risk management measures, (iii) the costs (to compare against the benefits) of different adaptation strategies, (iv) storms that occur on larger, regional, spatial scales to understand wider disruption impacts. This will provide the basis for a more comprehensive approach to appraise the benefits of adaptation of infrastructure in the urban environment. Ultimately, the methodology could be adapted to explore other hazard impacts, different types of infrastructure networks and potentially cascading failures between infrastructure systems [33-34].

\section{Acknowledgments}

The authors would like to acknowledge their funders for enabling this research to take place: Pregnolato is funded by a Doctoral Training Account (DTA) funded by EPSRC (EP/L504828/1), Ford is funded by the European Community's Seventh Framework Programme under Grant Agreement No. 308497 RAMSES (Reconciling Adaptation, Mitigation and Sustainable dEvelopment for citieS) project; Dawson is funded by an ESPRC fellowship (EP/H003630/1). They would also acknowledge Ray King at the UTMC in Newcastle upon Tyne for his assistance with access to travel data, Ian Abernethy at Gateshead City Council for access to the TADU system, and Vassilis Glenis and Robert Bertsch for their assistance with CityCAT modelling.

\section{References}

1. Evans-Pughe C. (2016). What can we do to hold back the waters? Engineering \& Technology, 2, 26-31.

2. Zio E. (2014). Vulnerability and Risk Analysis of Critical Infrastructures, Vulnerability, Uncertainty, and Risk, 23-30.

3. Hernandez-Fajardo I. and Dueñas-Osorio L. (2013). Probabilistic study of cascading failures in complex interdependent lifeline systems. Reliability Engineering \& System Safety, 111(0), 260-272.

4. Swiss RE (2014). Natural catastrophes and man-made disaster in 2013. Executive Summary.

5. Pant R., Hall J., Barr S. and Alderson D. (2014). Spatial Risk Analysis of Interdependent Infrastructures Subjected to Extreme Hazards, Vulnerability, Uncertainty, and Risk, 677-686.

6. Sayers P.B., Walsh C. and Dawson R. J. (2015). Climate Change Risk Assessment 2017: Projections of future flood risk in the UK. Committee on Climate Change, London.

7. Dunnett N.P. and N. Kingsbury (2004). Planting Green Roofs and Living Walls, Timber Pres, Portland (OR). 
8. Houghton J., Reiners J. and Lim C. (2009). Intelligent transport: how cities can improve mobility. IBM, New York (USA).

9. Demuzere M., Orru K., Heidrich O., Olazabal E., Geneletti D., Orru H., Bhave A. G., Mittal N., Feliu E. and Faehnle M. (2014). Mitigating and adapting to climate change: Multi-functional and multi-scale assessment of green urban infrastructure. Journal of Environmental Management, 146, 107-115.

10. Kilsby C., (2016). Seven ways to better manage flooding in the UK, The Conversation, accessed on February 4, 2016

11. Pitt M. (2008). The Pitt Review - Learning Lessons from the 2007 floods. Cabinet Office, London

12. Czemiel Berndtsson J. (2010). Green Roof Performance towards Management of Runoff Water Quantity and Quality: A Review. Ecological Engineering, 36(4), 351-360.

13. Ford A., Barr S., Dawson R. J. and James P. (2015). Transport Accessibility Analysis Using GIS: Assessing Sustainable Transport in London. ISPRS International Journal of Geo-Information, 4(1), 124149.

14. Hooper E., Chapman L. and Quinn A. (2012). Investigating the impact of precipitation on vehicle speeds on UK motorways. Meteorol. Appl., 21(2), 194-201.

15. Koetse M. J. and Rietveld P. (2009). The impact of climate change and weather on transport: An overview of empirical findings. Transportation Research Part D: Transport and Environment, 14(3), 205-221.

16. Suarez P., Anderson W., Mahal V. and Lakshmanan,T. R. (2005). Impacts of flooding and climate change on urban transportation: A systemwide performance assessment of the Boston Metro Area. Transportation Research Part D: Transport and Environment, 10(3), 231-244.

17. Tsapakis I., Cheng T. and Bolbol A. (2013). Impact of weather conditions on macroscopic urban travel times. Journal of Transport Geography, 28, 204-211.

18. Wright N. G., Thorne C. R. and Lawson E. (2014). Delivering and evaluating multiple flood risk benefits in blue-green cities. $11^{\text {th }}$ International Conference on Hydroinformatics (HIC 2014), New York (USA).

19. Kreibich H. and Thieken A. (2009). Coping with floods in the city of Dresden, Germany. Natural Hazards, 51(3), 423-436.

20. Merz B., Kreibich, H., Schwarze R. and Thieken A. (2010). Assessment of economic flood damage. Nat. Hazards Earth Syst. Sci., 11(6), 1697-1724.

21. Robson A. and Duncan R. (1999). Flood estimation handbook (v.3), Institute of Hydrology, Wallingford (UK).

22. Glenis V., McGough A. S., Vedrana K., Chris K. and Woodman S. (2013). Flood modelling for cities using Cloud computing. Journal of Cloud Computing, 2(1), $1-14$.

23. Stovin V.R. (2010). The potential of green roofs to manage urban stormwater. Water and Environment Journal, 24(3), 192-199.
24. Penning-Rowsell E., Priest S., Parker D., Morris J., Tunstall S., Viavattene, C., Chatterton J. and Owen D. (2013). Flood and coastal erosion risk management: A Manual for Economic Appraisal, Routledge.

25. Pregnolato M., Ford A., Wilkinson S., Dawson R. J. (in submission). The impact of flooding on urban transport sector - an integrated depth-disruption function. Journal of Transport Geography.

26. Galatioto F., Glenis V., Roberts R., Kilsby C. (2014). Exploring and modelling the impacts of rainfall and flooding on trnsport network. The case study of Newcastle upon Tyne. $2^{\text {nd }}$ International Conference on Urban Sustainability and Resilience (USAR 2014), University College London, London (UK).

27. Ong G.P. and Fwa T.F. (2008). Hydroplaning Risk Managment for Grooved Pavements. $7^{\text {th }}$ International Conference on Managing Pavement Assets, Calgary (Canada).

28. Chung, Y. and Recker W.W. (2012). A methodological approach for estimating temporal and spatial extent of delays caused by freeway accidents. IEEE Transactions, 13(3), 1454-1461.

29. Boyce D.(2012). Predicting road traffic route flows uniquely for urban transportation planning. Studies in Regional Science, 42(1), 77-91.

30. Dijkstra E.W. (1959). A note on two problems in connexion with graphs. Numerische Mathematik, 1(1), 269-271.

31. Dalziell E. and Nicholson A. (2001). Risk and Impact of Natural Hazards on a Road Network. Journal of Transportation Engineering, 127(2), 159-166.

32. Larsen M., Nielsen N. H. and Rasmussen S. F. (2010). The blue spot model. Development of a screening to assess flood risk on national roads and highways system. Report no. 23, Road Directorate, Danish Road Institute, Copenaghen (Denmark).

33. Ford A., Jenkins K., Dawson R. J., Pregnolato M., Bar, S., Hall J. (2014). Simulating Impacts of Extreme Weather Events on Urban Transport Infrastructure in the UK. International Symposium for Next Generation Infrastructure (ISNGI 2014), Wien (Austria).

34. Fu, G., Dawson, R. J., Khoury, M. and Bullock, S. (2014) Interdependent networks: Vulnerability analysis and strategies to limit cascading failure, European Physical Journal Part B, 87(7):148 